\title{
Research on Secure Network Coding in Wireless Sensor Networks Based on Web of Things Service
}

\author{
Zou Donglan ${ }^{*}$
}

College of Mathematics and Computer, Xinyu University, JiangXi, China

\begin{abstract}
In wireless sensor networks, clustering is a kind of effective topology control method. It has a clear hierarchical structure, load balancing, network reliability, strong expansibility and robustness. Currently, there have problems in some clustering protocols, such as the clusters distribute unevenly, the selection of cluster heads does not consider the residual energy and the communication distance deliberately, and the state of clustering does not change with the change of the network states. In order to resolve these problems, we design a Virtual Coordinates Dynamic Adaptive Clustering protocol (VCDAC). The protocol divides the network into clusters according to the idea of virtual partition and decides the number of clusters according to the network states, so that the energy consumption is reduced. The simulation results show that the protocol can prolong the network lifetime significantly, balance the network load, and send more data to the base station.
\end{abstract}

Keywords: Virtual coordinates dynamic adaptive clustering, Network coding, Internet of things, Web of things service, Wireless sensor networks.

\section{INTRODUCTION}

The theory of network coding was established by R. Ahlswede, N. Cai, S. Y. R. Li, and R. W. Yeung in around 2000 [1]. This result is an important extension of C. E. Shannon's point to point communication theory to complex networks [2]. The in-node signal processing in modern communication system is becoming more and more cheaper while the finite frequency spectrum resources are facing more and more communication demands. Network coding theory can be viewed as exchanging the in-node computation cost for network throughput, which immediately attracts lots of attentions since its birth. However, there are two big difficulties in putting the beautiful theory into practice. First, practical networks are significantly different from the ideal model used in establishing network coding theory. If those practical issues cannot be well solved, the throughput gain from network coding will be limited. Second, linear network coding explicitly assumes that the underlying supporting network is lossless. It is a challenging task to apply the theory into wireless environment, due to the fading and the broadcasting characters of wireless channels [3, 4].

Being a revolutionary breakthrough in the field of communication, network coding breaks the conventional wisdom of information transmission and processing, and has remarkable significance in communication systems. Network coding expands the traditional routing mechanism that a muter can only simply store and forward information, and the node in communication network can encode and decode the

\footnotetext{
*Address correspondence to this author at the College of Mathematics and Computer, Xinyu University, JiangXi, 338004, China;

E-mail: Zoudonglan@xyu.edu.cn
}

information. Network coding that has been proved can approach the max-flow of multicast network, and can increase the network capacity and the utilizing efficiency of bandwidth effectively. For the significant potentials, network coding has received much concern in both academia and industrial community. As research continues, the network coding theory becomes more complete, and the applications of network coding have been expanded to P2P network, wireless network, network security, and network tomography. Network coding is relatively new theory and a newly emerging technology, though much research has been done in the application of network coding and some important research results have been achieved. Network coding is faced with many challenges, and there are a lot of key questions to answer [5].

Network coding allows the relay nodes to code the received data which is sent out by different source nodes, and then forwards the coded data to the destination nodes. According to the established protocol, the destination nodes decode the coded data into some original information, so they can obtain their data. Network coding can improve the system throughput and network bandwidth. Researching on wireless network coding has important implications for the development and promotion of this technology, as well as carrying out the follow-up study applications. In practical applications of the wireless network, since the signal has the characteristics of multicast, other nodes within the broadcast range of each node, can receive the message that node sends out [6]. Therefore, a series of data information will be coded to a packet [7]. Transmitting the same amount of data can contain more useful information. The transmission may burden more available information to raise channel utilization and network throughput. 


\section{THE FRAMEWORK OF NETWORK CODING}

Many applications based on Internet need to use the large-scale data delivery mechanism, such as IP multicast and P2P cooperative content distribution, to deliver information to a large number of users. How to obtain the better delivery performance with the limited network resources has become a "hot spot" in the research community of network information flow. To achieve the goal, network coding, as one of the most important breakthroughs on the theory of information processing and transmission, was proposed in recent years. The main principle behind network coding is that the relay (intermediate) nodes in the communication network make encoding and decoding operations to the data bits. Network coding generalizes the traditional data delivery mechanism where the data bits can only be stored and forwarded. With network coding, the communication network can achieve better transmission performance, such as higher network throughput and lower bandwidth consumption etc. Due to its significant potentials, network coding has received much concern in both the research and industrial community. Lots of famous universities and research agencies have put much attention on the research of network coding and finished some important works.

Network coding is a promising technology to throughput and reliability. The core of network coding is to encode received data at intermediate nodes between the source and the destination. With the assistance of encoding at intermediate nodes, we can store and encode incoming messages, which is different from the traditional coding forwarding only operation of an intermediate node. Except for improving throughput and robust, network coding can also enhance security and balance of network. Therefore, there are many applications based on network coding from wire to wireless. However, there are many problems unsolved before practical application.

First, random network coding improves practice of network coding. But it makes a trade-off between practice and decoding probability. Node can recover the original data with high probability as long as the size of finite field is big enough. In this paper, we research decoding probability equation on random network coding, and infer how to improve the decoding probability in small size of finite field. Second, because of combination of packets at intermediate nodes, the feedback scheme is different from traditional network. Therefore, we research the problem on how to feedback in the environment of random network coding and discuss basically the disciplines of feedback scheme in network coding application. At last, there are new challenges on security of network employed network coding. In this paper, we researched the security schemes of network coding against pollution attack and wiretapping attack which are the main threats to security of network.

Building on re-transmission model and introducing many factors, we propose a new, more accurate than before and intact decoding probability equation of random network coding. According to numerical evaluation, we found the influence pattern on decoding probability of the size of finite field, network capacity, the size of generation, receiving capacity and transmission times. Besides, we give low bound of redundancy packets under different finite field. We propose orthogonal feedback scheme for network coding and give a fast algorithm of creating orthogonal codes in any finite field and dimension. According to our simulations, we prove that the orthogonal feedback scheme is available and shows better and stable performance than others in different topologies. To provide error correction, pollution inspection and resistance to wiretap at the same time, we propose two security schemes based on rank-metric code.

It can be proved from the theoretical aspects that network coding can enhance the performance of a given data network to a large extent. However, compared with the traditional data delivery mechanism based on storing and forwarding, the nodes in network coding system need to perform encoding and decoding operations such that the system based on network coding takes additional computational overheads (such as I/O and CPU consumption), which will bring high cost (computational and non-computational) to deploy network coding in real data networks. Hence, it is better to find out an optimal network coding scheme in which the additional computational overheads caused by network coding are as low as possible.

Information flow vector-base optimal network coding model. By modelling the information transmitted in the data delivery network as network information flow, we present a simple but effective approach to describe the information flow on each link in the data delivery system, referred to information flow vector. We analyze the properties of information flow vector, and conclude some requirements the information flow vector should meet with, and then construct an optimal network coding model. With the model, the problem of reducing the additional computational overheads of network coding operations can be converted into a programming formulation. Therefore, we can find out the optimal network coding-based data delivery scheme with minimal computational overheads by solving the model. Moreover, we consider some special cases and proposed a utility trade-off-based network coding model in which the utility and computational overheads are considered together. From the viewpoint of practical interests, we analyze the inherent characteristics of network coding and show that the essence of network coding is that there are some key links shared by deferent paths between the source and multi-receivers when we establish the transmission paths for the data delivery network.

To achieve the maximal throughput of the network, network coding is needed and the key links should carry encoded information. Since the additional computational overheads in network coding system are caused by the network coding operations, an effective way of reducing additional computational overheads and realizing a low-cost network coding transmission scheme is to reduce the number of key links while constructing transmission paths. Following this idea, we present a key links-based algorithm for low-cost network coding and propose its distribution implementation. Cluster network coding-based content distribution scheme. 
Due to the technical and non-technical reasons, the IP multicast based data delivery system which the network coding is originally proposed for, has not been widely implemented in the network till now. As a scale solution to deliver content to a large number of end-users, the overlay network based P2P cooperative data delivery scheme and systems have been widely used recently. Borrowing the idea of network coding, a novel data delivery scheme which is based on network coding was proposed. However, some researchers think the performance of such novel scheme be less optimistic due to the additional computational overheads caused by network coding operations. To optimize the data delivery scheme and enhance the performance further, we proposed some new mechanisms, such as clustered network coding, random choice-based strategy and independence checking algorithm etc. Based on the proposed mechanisms, we construct an improved network coding-based data delivery scheme. Compared to the commonly used data delivery scheme, the improved scheme can reduce the additional computational consumptions of network coding operations to a large extent, and improve the performance of data delivery system significantly.

\section{SECURE NETWORK CODING BASED ON WEB OF THINGS SERVICE}

The emergence of the Internet of Things has its specific historical background. The economic weakness of the western developed countries and growth worry in developing countries are the internal demands of the Internet of Things. The sub-prime mortgage crisis originated in the United States in 2008 is the fire cable of the rapid growth of the Internet of Things. In order to promote the development of the Internet of Things, some developed countries carry scientific strategic layout on it based on its national interests. Research on the Internet of Things of our country is late, because we have launched a series of resource mobilization activities and local industrial layout on the background of not grasping accurately connotation and epitaxial of the Internet of Things, growth process, the development directions, which is not conducive to the healthy development of the Internet of Things Industry in China. Based on these, it will be helpful to improve the progress of the theory of the Things on Internet industry, and also be conducive to the manager's scientific decision-making of the Internet of Things industry in China, to study systematically domestic and foreign related base theory of the Internet of Things and base theory of industry growth, to analyze the growth mechanism of the Internet of Things industry, and to explore its growth law.

The Internet of Things is a product of IT and Internet's development to a certain stage, and is a new emerging modern information industry. However, from the content, the Internet of Things is different from the general information industry. It includes not only sensor identification equipment, network communications equipment, application system equipment and so on other physical products manufacturing industry, but also responsible for providing communication channels, software development, system integration, professional services and so on other service industries, or the spirit of industry. It not only has some new creation, newly added products "narrow" industrial part of the Things of Internet technology itself, but also has some newly added "fusion" industrial part by application extending to other industries. The Things of Internet has a complex industrial connotation and extension. The theory of growth mechanism of the Things of Internet industry is based on the theory of information industry, the theory of core firm, the theory of Industry chain and the theory of industry cluster.

The Internet of Things, like other industry, has also an evolutionary process on industry growth, only it shows some differences in growth background, power source, the growth process and so on. The value creation of the Internet of Things is the innovative way of communication between humans and things, to get real raw data, to promote transparency of the management process, and reduce the communication transaction costs. Mainly, the show of its value creation process is to promote simplistic data access, promote the automation of production processes and management, and enhance customer service levels. The application value of the Internet of Things is mainly showed to make the object wise, and to change the mode way of production and life of the human.

In Wireless Sensor Networks, clustering is a kind of effective topology control method. It has clear hierarchical structure, load balancing, network reliability, strong expansibility and robustness. Currently, there have problems in some clustering protocols, such as the clusters distribute unevenly, the selection of cluster heads does not consider the residual energy and the communication distance deliberately, and the state of clustering does not change with the change of the network states. In order to resolve these problems, we design a Virtual Coordinates Dynamic Adaptive Clustering protocol (VCDAC). The protocol divides the network into clusters according to the idea of virtual partition and decides the number of clusters according to the network states, so that the energy consumption is reduced. The simulation results show that the protocol can prolong the network lifetime significantly, balance the network load, and send more data to the base station.

Topology control has a great influence on the performance of Wireless Sensor Network. Excellent topology can improve the efficiency of the routing protocol and MAC protocol, and provide help for target localization, time synchronization and data fusion. Cluster-based network is a clear hierarchical topology control method, it can prolong the network lifetime, and often formulate into optimization problem to find the optimal solution. Conventional analysis methods need tremendous computation, which grows up exponentially with the problem scale increase. But for implementation on a wireless sensor node, an optimization method that requires moderate or even minimal computing and memory resources and yet produces better results is desirable. Particle Swarm Optimization is a computationally efficient bio-inspired optimization method compared to traditional analytical methods. 


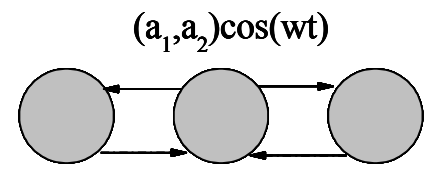

$$
\mathrm{a}_{1} \cos (\mathrm{wt}) \quad \mathrm{a}_{2} \cos (\mathrm{wt})
$$

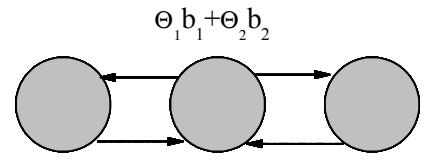

$\Theta_{1} b_{1} \quad \Theta_{2} b_{2}$

Physical Layer Network Code $-\cdots-\cdot-1 \longrightarrow 2$ Complex Domain Network Code

A

B

Fig. (1). The network coding of relay nodes in the physical layer and complex field.

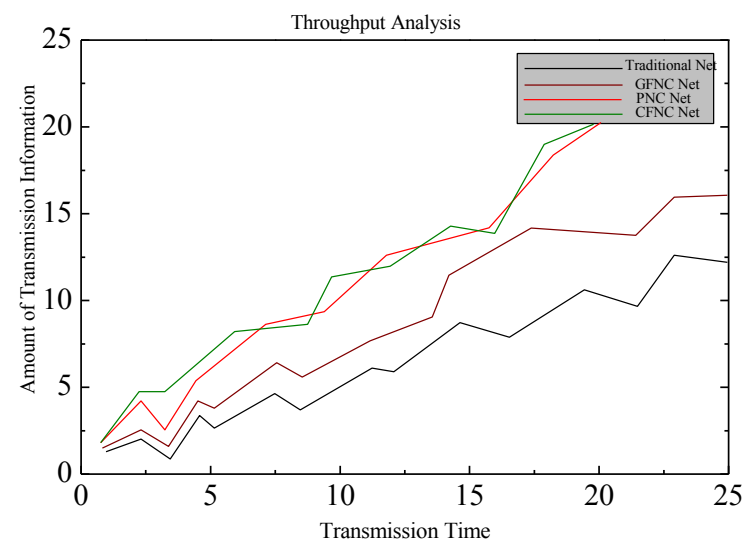

Fig. (2). Throughput of four kinds of methods between two nodes.

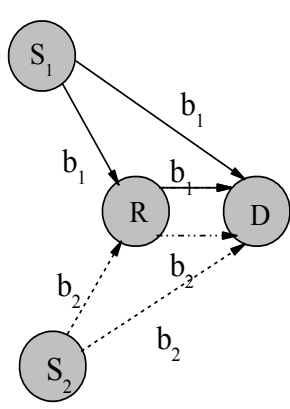

(a)Traditional relay

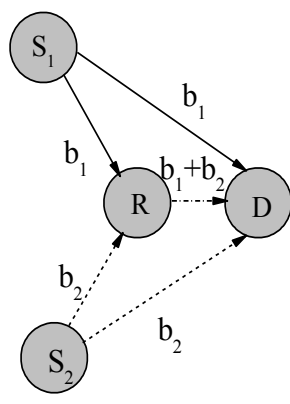

(b)GFNC relay

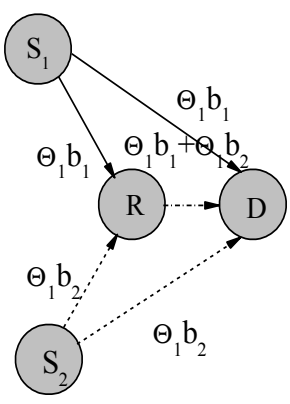

(c)CFNC relay

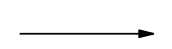

1 3

Fig. (3). Wireless relay network.

\section{TWO NODES INFORMATION TRANSMISSION MODEL}

Two nodes model and the network coding of relay nodes in the physical layer and complex field are shown in Fig. (1).

It can be seen through the curve of exchange information as long transmission time as shown in Fig. (2). Fig. (2) also shows the analyses and comparison of the curve of the throughputs of four methods when the information of two nodes is exchanged.

In order to make understanding and analysis intuitive, simulations are carried on the calculation of the three relay methods as shown in Fig. (3). It can be seen through the 


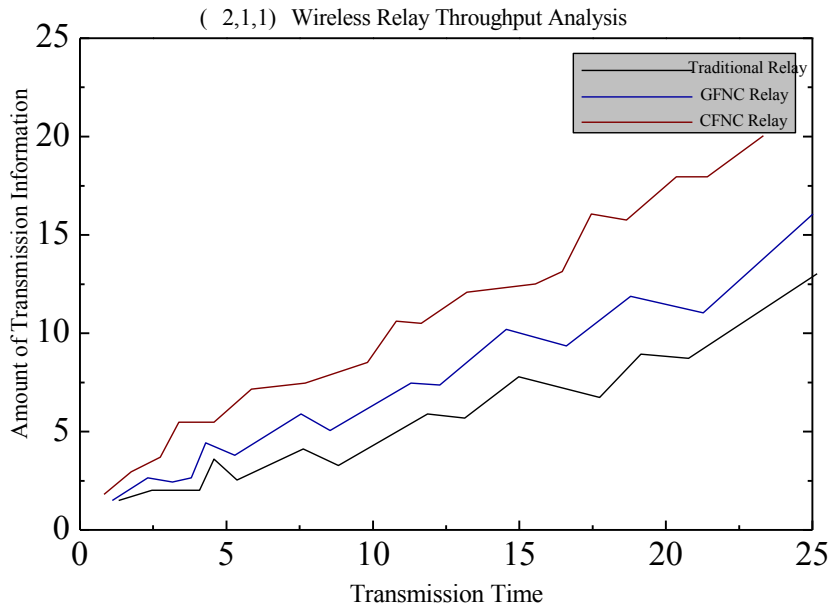

Fig. (4). Throughput of three kinds of wireless relay methods.

curve of exchange information as long transmission time as shown in Fig. (4). Along with the change of transmission time, the throughput of CFNC relay method is better than that of GFNC relay method. The throughput of GFNC relay method is also better than that of traditional relay method.

\section{CONCLUSION}

Due to most researchers mainly focusing on coding algorithms and performance evaluation and application scheme for network coding, a physical layer network coding scheme realized by symbol mapping is proposed in the paper, and the impact of the modulation and power allocation on bit error rate and outage probability is also comparatively analyzed. In order to resolve these problems, we design a Virtual Coordinates Dynamic Adaptive Clustering protocol, which divides the network into clusters according to the idea of virtual partition and decides the number of clusters according to the network states, so that the energy consumption is reduced. The simulation results show that the protocol can prolong the network lifetime significantly,

\section{CONFLICT OF INTEREST}

The author confirms that this article content has no conflict of interest.

\section{ACKNOWLEDGEMENTS}

Declared none.

\section{REFERENCES}

[1] J. Yick, B. Mukherjee, and D. Ghosal, "Wireless sensor network survey," vol. 52, no. 12, pp. 2292-2330, 2008.

[2] K. Karenos, U. Kalogeraki, and S. Krishnamurthy, "Cluster-based congestion control for sensor networks", ACM Transactions on Sensor Networks, vol. 4, pp. 1-39, 2008.

[3] R. V. Kulkarni, and G. K. Venayagamoorthy, "Particle Swarm Optimization in Wireless Sensor Networks: A Brief Survey," IEEE Trans. Systems, Man, and Cybernetics, vol. 41, no. 2, pp. 262-267, 2011.

[4] X. Wang, and J. Zhang, "Iot research review," Journal of Liaoning University, vol. 3, pp. 214-217, 2010.

[5] S. Shen, Q. Fan, and P. Zong, "Research on the architecture and related technology of IOT," Journal of Nanjing University of Posts and telecommunications, no. 6, pp. 1-11, 2009.

[6] W. Liu, H. Wang, and Q. Xiao, "The distinction of IOT conception," Journal of Telecommunications Technology, no. 1, pp. 5-8, 2010.

[7] N. Latiff, C. Tsimenidis, and B. Sharif, "Performance comparison of optimization algorithms for clustering in wireless sensor networks," In: Proc. IEEE Int. Conf. Mobile Ad Hoc Sens. Syst., vol. 1, pp. 1-4, 2007.

(C) Zou Donglan; Licensee Bentham Open.

This is an open access article licensed under the terms of the Creative Commons Attribution Non-Commercial License (http://creativecommons.org/licenses/by$\mathrm{nc} / 4.0 /$ ) which permits unrestricted, non-commercial use, distribution and reproduction in any medium, provided the work is properly cited. 